\title{
Substrate specificity of aquatic extracellular peptidases assessed by competitive inhibition assays using synthetic substrates
}

\author{
Andrew D. Steen ${ }^{1, *}$, Jasmine P. Vazin ${ }^{2}$, Shane M. Hagen ${ }^{3}$, Katherine H. Mulligan ${ }^{2,4}$, \\ Steven W. Wilhelm ${ }^{2}$ \\ ${ }^{1}$ Department of Earth and Planetary Sciences, University of Tennessee, 306 EPS Building, Knoxville, TN 37996, USA \\ ${ }^{2}$ Department of Microbiology, University of Tennessee, M409 Walters Life Sciences, Knoxville, TN 37996, USA \\ ${ }^{3}$ Department of Civil and Environmental Engineering, University of Tennessee, 325 John D. Tickle Building, Knoxville, \\ TN 37996, USA \\ ${ }^{4}$ Department of Biology, University of North Carolina at Chapel Hill, Coker Hall, CB \#3280, Chapel Hill, NC 27599, USA
}

\begin{abstract}
The identities and biochemical properties of extracellular enzymes present in natural environments are poorly constrained. We used a series of competitive inhibition experiments with samples from a freshwater environment (the Tennessee River at Knoxville, TN, USA) and a marine environment (Bogue Sound, NC, USA) to characterize the range of substrate specificities of naturally occurring enzymes that hydrolyze L-leucine 7-amido-4-methylcoumarin (Leu-AMC), L-proline-AMC (Pro-AMC), and L-arginine-AMC (Arg-AMC) - putative substrates for leucylaminopeptidase, prolyl-aminopeptidase, and arginyl-aminopeptidase, respectively. Extracellular peptidases which hydrolyzed Arg-AMC and Leu-AMC demonstrated affinity for up to 8 other amino acids, whereas those hydrolyzing Pro-AMC in the Tennessee River, and Arg-AMC at Bogue Sound, were more specific to proline and arginine, respectively. Patterns of substrate affinity showed that Leu-AMC (at both sampling sites) and Arg-AMC (at Bogue Sound) were primarily hydrolyzed by enzymes other than leucyl-aminopeptidase and arginyl-aminopeptidase, respectively. The set of naturally occurring peptidases in both environments showed greater affinity towards a subset of amino acids. These amino acids were on average larger, yielded more free energy from oxidation to $\mathrm{CO}_{2}$, and tended to be depleted in aged organic matter. These relationships indicate that pathways of amino acid diagenesis are at least partially controlled by the substrate specificities of the peptidases involved in protein degradation.
\end{abstract}

KEY WORDS: Extracellular enzymes $\cdot$ Peptidases $\cdot$ Protein degradation $\cdot$ Heterotrophy $\cdot$ Amino acids

\section{INTRODUCTION}

Extracellular enzymes mediate the transfer of macromolecular organic carbon into the microbial loop. Investigations of the identities and activities of extracellular enzymes, as well as the controls on their function in aquatic environments, have therefore been seen as a key step towards understanding the controls on organic matter reactivity in aquatic ecosystems (Arnosti et al. 2014). Biochemists frequently

${ }^{*}$ Corresponding author: asteen1@utk.edu study enzyme function by extracting specific enzymes from tissue and measuring their properties in isolation. This approach has not been successful for environmental enzymes, however; purifying extracellular enzymes from environmental samples is analytically challenging because these enzymes are highly diverse (Arrieta \& Herndl 2002, Obayashi \& Suzuki 2005) and are present in low concentrations relative to a large background of organic matter. Moreover, it is likely that efforts to isolate these enzymes would 
alter their activities and functional characteristics (Wang et al. 2012). Environmental studies of extracellular enzymes are therefore typically restricted to measurements of the hydrolysis rates of added substrates that are meant to mimic macromolecules presumed to be present in the environment. These substrates may be unlabeled macromolecules (Liu et al. 2010) or macromolecules labeled with either fluorophores (Arnosti 1996, Pantoja et al. 1997, Pantoja \& Lee 1999) or spin labels (Steen et al. 2006), which allow analysis by election paramagnetic resonance spectroscopy. Most frequently, however, they are small fluorogenic substrate proxies (Hoppe 1983, German et al. 2011), which consist of a small biologically relevant monomer or oligomer covalently bound to a fluorophore. Fluorescence of the compound is quenched until the fluorophore-biomolecule bond is cleaved. Enzyme activity is subsequently inferred from the rate of increase of fluorescence in the sample and is expressed in terms of the rate at which free fluorophore is liberated.

This method of assessing extracellular enzyme activity is rapid, sensitive, and inexpensive. However, interpretation of the resulting data is complicated by the fact that hydrolytic enzymes have variable, and sometimes broad, substrate specificities (Nomoto et al. 1960). Hydrolysis of, for instance, L-leucyl-7-amido-4-methylcoumarin (Leu-AMC) is often interpreted as the potential activity of a single specific enzyme, leucyl-aminopeptidase, EC 3.4.11.1 (e.g. Steen \& Arnosti 2013), but this interpretation is not necessarily correct. Leu-AMC hydrolysis could also represent the concerted action of many distinct enzymes, each of which may display some activity towards N-terminal Leu, but preferentially hydrolyze different residues. Enzyme assays based on unlabeled (Liu et al. 2010) or fluorescently labeled oligopeptides (Pantoja et al. 1997, 2009, Pantoja \& Lee 1999) have shown that amino acid sequence strongly influences hydrolysis rates of oligopeptides. The few studies which report hydrolysis rates of small fluorogenic peptidase proxies other than Leu-AMC show that different peptidase substrates may be hydrolyzed at very different rates (Obayashi \& Suzuki 2005, 2008b, Steen \& Arnosti 2013). Substrate specificities of peptidases expressed by pure cultures of marine bacteria have been investigated (Odagami et al. 1994), but the substrate specificities of the mix of peptidases naturally present in aquatic samples is unknown. It is therefore not clear how to correctly interpret the hydrolysis rates of multiple small substrate proxies measured in parallel on the same sample; they could reflect the activities of multiple, highly substrate-specific enzymes, or they could reflect overlapping activities of substrate-unspecific enzymes (Fig. 1). This distinction is important for the interpretation of small substrate proxy-based enzyme assays. If each small-substrate proxy represents the activity of a distinct, substrate-specific enzyme, the total environmental peptidolytic potential is best approximated by the sum of $V_{\max }$ values of many small-substrate proxies. Conversely, if enzymes are generally substrate nonspecific, the highest individual $V_{\max }$ measured most closely approximates the total environmental peptidolytic potential.

The goal of this project was to quantitatively determine the substrate specificities of several aminopeptidases in a freshwater environment (the Tennessee River at Knoxville, TN, USA) and a marine environment (Bogue Sound, NC, USA). These peptidases are likely derived from a range of sources including prokaryotes as well as protists (Thao et al. 2014). To accomplish this, we determined the affinity of 12 amino acid- $p$-nitroanilide compounds (Xaa-pNA) for the naturally occurring enzymes hydrolyzing 3 fluorogenic peptidase substrates: L-leucine-AMC (Leu-AMC), Larginine-AMC (Arg-AMC), and L-proline-AMC (ProAMC), which are substrates for leucyl-, arginyl- (EC 3.4.11.6), and prolyl-aminopeptidase (EC 3.4.11.5), respectively. (In order to maintain a simpler kinetic model, we only examined activities of aminopeptidases rather than endopeptidases such as trypsin or chymotrypsin). We determined these affinities by measuring the extent to which each Xaa-pNA competitively inhibited the hydrolysis of each AMC substrate. These competitive inhibition experiments revealed the functionality of the set of enzymes that hydrolyze each small fluorogenic substrate proxy, without requiring isolation or purification of those enzymes.

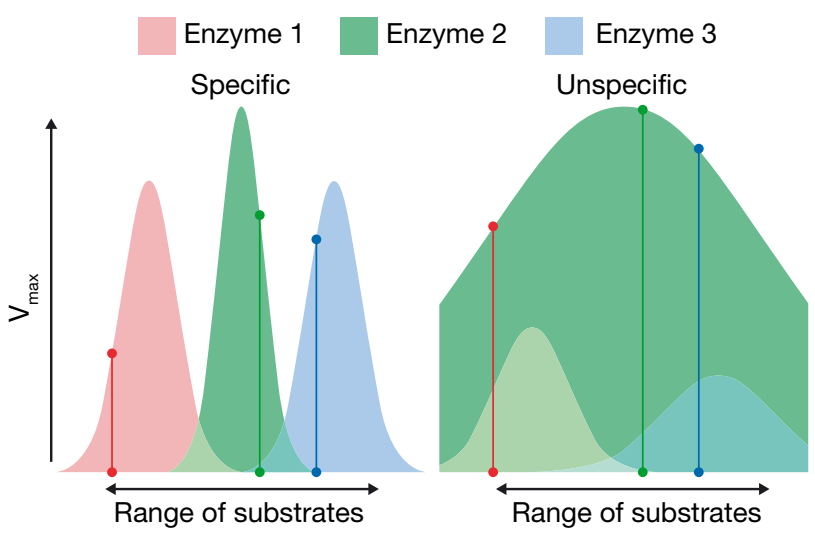

Fig. 1. Conceptual illustration of overlapping enzyme substrate specificity and possible interpretations of fluorogenic substrate hydrolysis rate measurements. $V_{\text {max }}$ : maximum theoretical hydrolysis rate 


\section{MATERIALS AND METHODS}

\section{Measurements of substrate hydrolysis rate $v_{0}$}

Arg-AMC, Leu-AMC, and Pro-AMC (collectively Yaa-AMC) hydrolysis rates were measured in the Tennessee River at the Volunteer Landing dock in Knoxville, TN, and in Bogue Sound from the University of North Carolina Institute of Marine Sciences Dock in Morehead City, NC, on the dates listed in Table 1. Surface water samples were collected by bucket and immediately transported to the laboratory (<10 min transit time). Hydrolysis rates were measured in unfiltered water at room temperature $(22 \pm$ $0.5^{\circ} \mathrm{C}$ ). For each combination of Xaa-pNA substrate, Xaa-pNA concentration, and Yaa-AMC substrate, $1 \mathrm{ml}$ of unfiltered sample was added to a $2 \mathrm{ml}$ methacrylate cuvette and amended with buffer at approximately in situ pH (Tennessee River: $100 \mathrm{mM}$ phosphate buffer, $\mathrm{pH}=7.6$; Bogue Sound: artificial seawater made from Sigma-Aldrich sea salts, salinity $=30, \mathrm{pH}=8.2)$ plus $16 \mu \mathrm{l}$ AMC substrate $(10 \mathrm{mM}$ stock, in DMSO [dimethyl sulfoxide], for a concentration of $138 \mu \mathrm{M})$, plus between 0 and $36 \mu \mathrm{l}$ of Xaa-pNA (10 mM stock, for a final concentration of 0 to $314 \mu \mathrm{M}$ ), plus a volume of DMSO (0 to $36 \mu \mathrm{LMSO}$ depending on the volume of Xaa-pNA stock added) such that the final quantity of DMSO in all samples was equal (3.4\% DMSO v/v). Cuvettes were capped and mixed prior to incubation.

Fluorescence was measured using a Quanti-Fluor $\mathrm{ST}$ solid state fluorometer set to the ' $U V^{\prime}$ mode. For each cuvette, fluorescence was measured 4 to 6 times over the course of a 2 to $4 \mathrm{~h}$ incubation. In all cases, the relationship between fluorescence and time was linear, indicating that production or degradation of enzymes during the experiment was negligible. Temperature and the fluorescence of a standard were monitored every ca. 3 min (Steen \& Arnosti 2011) to assess the stability of fluorescence measurements; both temperature and instrument response were stable during the course of each incubation.

Table 1. Sampling dates (in 2013) and in situ water temperatures. AMC: 7-amido-4-methylcoumarin

\begin{tabular}{|lccccc|}
\hline \multirow{2}{*}{ Yaa-AMC } & \multicolumn{2}{c}{ Tennessee River } & \multicolumn{2}{c|}{ Bogue Sound } \\
& Date & Temp. & \multicolumn{2}{c|}{ Date } & Temp. \\
\hline Arg-AMC & $7 \mathrm{Mar}$ & $8^{\circ} \mathrm{C}$ & $25 \mathrm{Mar}$ & $12^{\circ} \mathrm{C}$ \\
Leu-AMC & $10 \mathrm{Feb}$ & $9^{\circ} \mathrm{C}$ & $26 \mathrm{Mar}$ & $12^{\circ} \mathrm{C}$ \\
Pro-AMC & $21 \mathrm{Feb}$ & $9^{\circ} \mathrm{C}$ & & $26 \mathrm{Mar}$ & $12^{\circ} \mathrm{C}$ \\
\hline
\end{tabular}

\section{Competitive inhibition experiments}

Kinetics of hydrolytic enzymes may be described by the Michaelis-Menten equation such that:

$$
v_{0}=\frac{V_{\max }[S]}{K_{\mathrm{m}}+[S]}
$$

where $V_{0}$ is the observed hydrolysis rate, $V_{\max }$ is the maximum theoretical hydrolysis rate, $[S]$ is the substrate concentration (here, Yaa-AMC), and $K_{\mathrm{m}}$ is the half-saturation constant. While environmental hydrolases do not always obey Michaelis-Menten kinetics (Steen \& Ziervogel 2012), each fluorogenic substrate exhibited Michaelis-Menten kinetics at each site in this study.

In the presence of a competitive inhibitor (XaapNA), $K_{\mathrm{m}}$ is reduced by a factor of $1+[I] / K_{\mathrm{I}}$, where $[I]$ is the concentration of the inhibitor and $K_{\mathrm{I}}$ represents the affinity between the enzyme active site and the inhibitor (a lower $K_{\mathrm{I}}$ represents a greater affinity) (Leskovac 2003); thus:

$$
V_{0}=\frac{V_{\max }[S]}{K_{\mathrm{m}}\left(1+\frac{[I]}{K_{\mathrm{I}}}\right)+[S]}
$$

In this experiment, $K_{\mathrm{I}}$ represents the affinity between a pure inhibitor (Xaa-pNA) and the mixed set of enzymes that hydrolyze a specific Yaa-AMC. Eq. (2) can be linearized with respect to $[I]$ by taking the inverse of each side, yielding:

$$
\frac{1}{V_{0}}=\frac{K_{\mathrm{m}}}{V_{\max } K_{\mathrm{I}}[S]}[I]+\frac{K_{\mathrm{m}}}{V_{\max }[S]}+\frac{1}{V_{\max }}
$$

We measured $K_{\mathrm{I}}$ by holding [S] constant and determining $v_{0}$ at inhibitor concentrations ranging from 0 to $314 \mu \mathrm{M} . K_{\mathrm{I}}$ can then be calculated from the slope $m$ of the relationship between $1 / v_{0}$ and $[I]$, with all other variables held constant, i.e.:

$$
K_{\mathrm{I}}=\frac{K_{\mathrm{m}}}{V_{\max }[S] m}
$$

Xaa-pNA substrates were chosen as competitive inhibitors for 2 reasons. First, they are considerably less expensive than custom-synthesized oligopeptides. Second, the pNA moiety is structurally more similar to AMC than to an amino acid, in that both compounds contain an aromatic ring and lack the same charge separation found in amino acids. Therefore, the kinetics of Yaa-AMC hydrolysis in the presence of Xaa-pNA are more influenced by differences in the N-terminal amino acid (Yaa vs. Xaa) than by differences in C-terminal moiety (pNA vs. AMC); differences in residue chemistry on the C-terminal side 
of the scissile bond might have dominated Yaa-AMC hydrolysis kinetics if we had used oligopeptides or proteins. In practice, hydrolysis rates of Xaa-pNA substrates are comparable to those of Yaa-AMC substrates in the same samples (Bailey et al. 2011).

In order to further isolate the effect of $\mathrm{N}$-terminal amino acid residue chemistry from the effect of the difference between AMC and pNA on the measured affinities, we expressed all affinities as $K_{\mathrm{I}} / K_{\mathrm{I}, \text { ref }}$, where $K_{\mathrm{I}, \text { ref }}$ is $K_{\mathrm{I}}$ for the pNA compound homologous to the AMC substrate, that is for Yaa-pNA. For instance, the $K_{\mathrm{I}}$ for Arg-pNA would be the $K_{\mathrm{I}, \text { ref }}$ for Arg-AMC. $K_{\mathrm{I}} / K_{\mathrm{I}, \mathrm{ref}}$ can then be calculated as:

$$
\frac{K_{\mathrm{I}, \text { Xaa }}}{K_{\mathrm{I}, \text { ref }}}=\frac{m_{\text {ref }}}{m_{\text {Xaa }}}
$$

with $m$ defined as in Eq. (4). This method has the further advantage of eliminating the error associated with imprecision in the measurements of $V_{\max }$ and $K_{\mathrm{m}}$. The limit of quantification for this method was calculated as the mean of the standard error of $m_{\text {Xaa }}$ divided by $m_{\text {ref }}$. This upper limit of quantification was measured to be 19.4. For all $m_{\text {Xaa }}$ which were not significantly greater than zero, $K_{\mathrm{I}} / K_{\mathrm{I}, \mathrm{ref}}$ was conservatively set to 19.4 .

\section{Verification of the kinetic model}

The kinetic model described in Eqs. (1) to (5) assumes a system consisting of a single, purified enzyme obeying Michaelis-Menten kinetics, a single substrate, and a single competitor. In environmental samples, multiple isofunctional enzymes were almost certainly present (Arrieta \& Herndl 2002). To validate this model for a system containing mixed, isofunctional enzymes, we simulated a system with 10 isofunctional enzymes. Each simulated enzyme had the same $V_{\max }$ but different $K_{\mathrm{m}}$ and $K_{\mathrm{I}}$ values. These conditions maximize the possibility of observing a divergence between the apparent simulated kinetics of the mixed enzymes and the kinetic model we employed. $K_{\mathrm{m}}$ values were randomly selected from a uniform distribution between 10 and $100 \mu \mathrm{M}$, and $K_{\mathrm{I}}$ values were randomly selected from a uniform distribution between 100 and $1000 \mu \mathrm{M}$. Defining $v_{\text {obs }}$ as the sum of $v_{0}$ that would be observed in this theoretical system of mixed enzymes, the linearity of $1 / v_{\text {obs }}$ as a function of $[I]$ was assessed (a linear relationship would indicate that the model approximations are reasonable).

Finally, we note that 2 types of natural inhibitors were likely present in our sample: protein-like material (i.e. peptidase substrate, which would act to com- petitively inhibit hydrolysis of Yaa-AMC) and potential nonspecific inhibitors such as humic substances. Since the concentration of these natural inhibitors was the same in each treatment, and was likely low relative to the concentration of added Xaa-AMC (up to $314 \mu \mathrm{M}$ ), the presence of these inhibitors was unlikely to substantially affect the results reported here. Finally, because we report all results as normalized relative to a $K_{\mathrm{I}, \text { ref }}$ (Eq. 5), the presence of inhibitors should not bias the results presented here.

\section{Correction for quenching by Xaa-pNA}

Xaa-pNA slightly quenches AMC fluorescence, likely due to weak optical absorbance by p-nitroanilide within AMC's excitation band. We quantified this effect by measuring fluorescence of $10 \mathrm{nM}$ AMC at Xaa-pNA concentrations varying from 0 to $314 \mu \mathrm{M}$, at pH 7.6 and at pH 8.2 (DMSO was added to each standard, such that the total volume of DMSO in each standard was $36 \mu \mathrm{l}$ regardless of the concentration of Xaa-pNA). This quenching was modeled with an exponential function, such that:

$$
f l_{\text {obs }}=f l_{0} \times \mathrm{e}^{-k[I]}
$$

where $f l_{\text {obs }}$ is the observed fluorescence in the presence of concentration $K_{\mathrm{I}}$ of Xaa-pNA and $f l_{0}$ is the corresponding unquenched fluorescence. The quenching coefficient $k$ was determined by nonlinear least-squares regression, and unquenched fluorescence values $\left(f l_{0}\right)$ were calculated based on observed fluorescence values $\left(f l_{\text {obs }}\right)$ and measured $k$.

\section{Evaluation of the effect of DMSO on enzyme kinetics}

To assess whether enzyme kinetics were influence by DMSO at the concentration used in this work $(3.4 \% \mathrm{v} / \mathrm{v})$, we measured saturation curves for LeuAMC hydrolysis in Tennessee River waters at DMSO concentrations between 0 and $5 \% \mathrm{v} / \mathrm{v}$ using a technique based on the recommendations of German et al. (2011). Up to $30 \mu \mathrm{l}$ Leu-AMC stock (2 mM LeuAMC dissolved in MilliQ- $\mathrm{H}_{2} \mathrm{O}$ without DMSO) were added to wells of a black 96-well plate. Then, 0 to $12.5 \mu \mathrm{l}$ DMSO were added to each plate, plus $25 \mu \mathrm{l}$ phosphate buffer $(0.2 \mathrm{M}, \mathrm{pH} 7.4)$ and a compensatory volume of MilliQ- $\mathrm{H}_{2} \mathrm{O}$ such that the combined volume of Leu-AMC stock, DMSO, and water totaled $62.5 \mu$ l. Finally, $162.5 \mu \mathrm{l}$ of unfiltered water from the 
Tennessee River, collected 2 June 2014, was added to each well. Fluorescence (excitation $=360 \mathrm{~nm}$, emission $=440 \mathrm{~nm}$ ) was measured every 5 min in a BioTek CytoSense 3 plate reader over the course of $1 \mathrm{~h}$. The incubation temperature was $26^{\circ} \mathrm{C}$. Hydrolysis rates were calculated as described at the start of the 'Materials and methods'.

\section{Data analysis}

All statistical analyses were performed using the $\mathrm{R}$ statistical platform (R Core Team 2013). Plots were created using the ggplot2 package (Wickham 2009). Where residuals were homoscedastic the correlations were assessed using linear least-squares models. When this was not the case, Spearman's $\rho$ was employed. An R package containing all raw data and functions to reproduce the analysis presented here is available at http://github.com/adsteen/subspec.

\section{RESULTS}

\section{Validation of the kinetic model}

Simulations showed that the inhibition kinetics of a mixed system of isofunctional enzymes, such as those that exist in aquatic environments, are nearly identical to the simplified system modeled in Eqs. (1) to (5). The root-mean-square deviation between the kinetics of the 10 simulated enzymes $\left(1 / v_{\text {obs }}\right)$ and modeled kinetics based on Eq. (3) was only $0.11 \%$ (Fig. 2), showing that Eqs. (1) to (5) constitute a reasonable
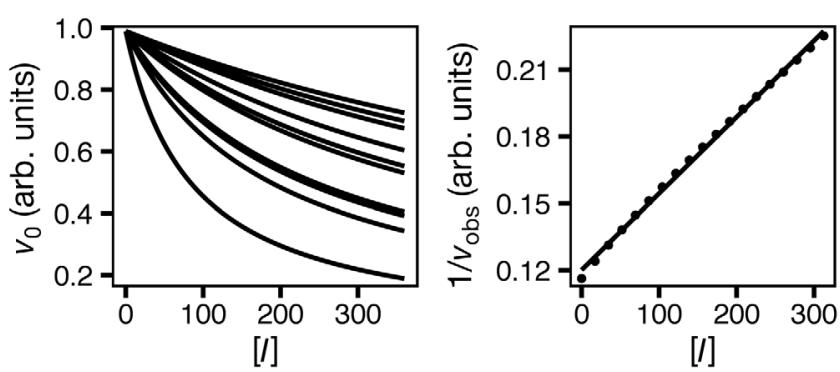

Fig. 2. Simulation of apparent kinetics arising from coexistence of 10 isofunctional enzymes, each with different $K_{\mathrm{I}}$ and $K_{\mathrm{m}}$. Left panel: simulated $v_{0}$ of each of 10 enzymes as a function of [I]. Right panel: $1 / v_{\text {obs }}$ for the 10 mixed, isofunctional enzymes as a function of $[I]$, compared to a linear fit through the data. Simulated data are plotted as points; the linear regression corresponding to Eq. (3) is represented as a solid line. $K_{\mathrm{I}}$ : affinity between enzyme site and inhibitor; $K_{\mathrm{m}}$ : halfsaturation constant; $v_{0}$ : hydrolysis rate catalyzed by each simulated enzyme; $[I]$ : concentration of inhibitor; $V_{\text {obs }}$ : sum of simulated $v_{0}$ values; arb.: arbitrary model for the competitive inhibition in the systems investigated here.

Hydrolysis of Pro-AMC in the Tennessee River was too slow to accurately measure $K_{\mathrm{I}}$, so those data are excluded from the subsequent discussion. Otherwise, uninhibited kinetics of substrate hydrolysis obeyed Michaelis-Menten kinetics (Fig. 3). Plots of $1 / v_{0}$ versus $[I]$ were generally linear at each site and for each substrate (Fig. 4), as predicted by Eq. (3), indicating that Xaa-pNA compounds inhibited Yaa-AMC hydrolysis via competitive inhibition rather than any other mechanism.

\section{Correction for fluorescence quenching by Xaa-pNA}

Fluorescence quenching of Yaa-AMC by Xaa-pNA empirically fit Eq. (6) (data not shown). Neither buffer $\mathrm{pH}$ nor the identity of the Xaa-pNA inhibitor significantly affected the fitted value of $k$, so a single $k$

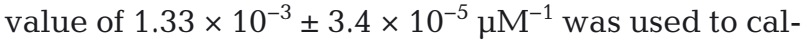
culate $f l_{0}$ in each experiment, based on Eq. (6) and observations of $f l_{\mathrm{obs}}$ and known $[I]$.

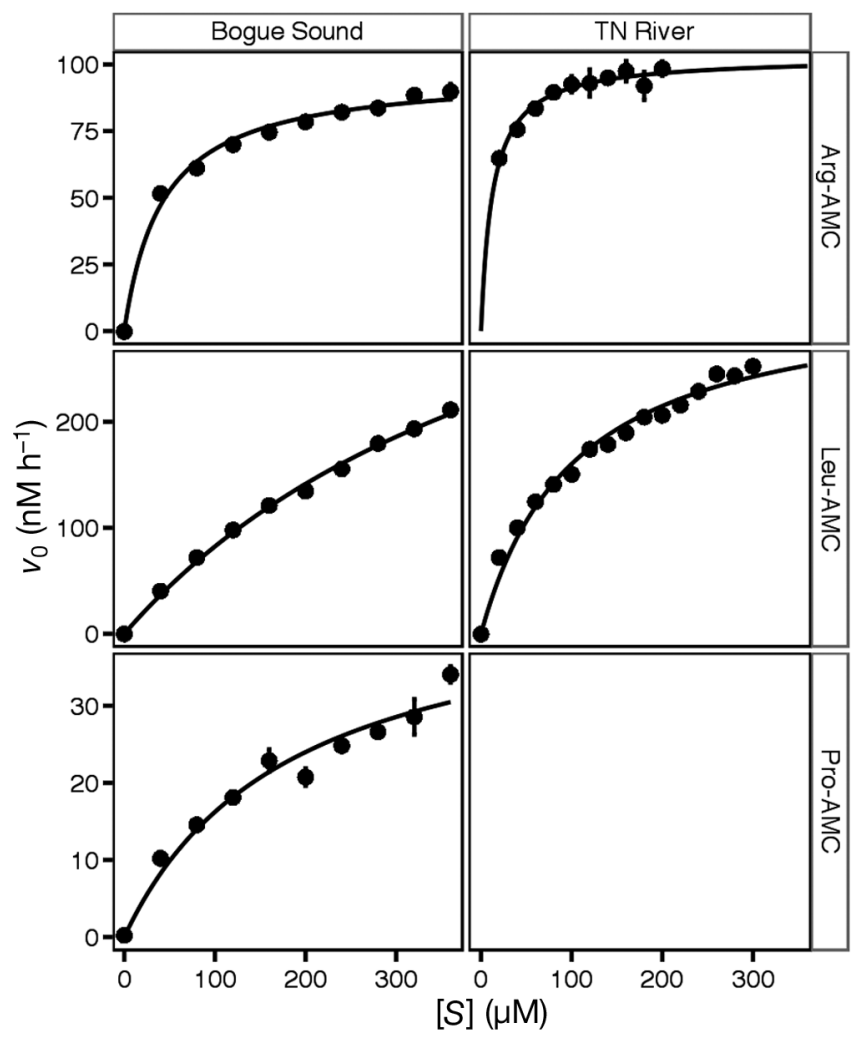

Fig. 3. Saturation curves for each substrate (see Table 1 for abbreviations). Pro-AMC was not detectable in the Tennessee (TN) River, so no saturation curve was measured. $v_{0}$ : observed hydrolysis rate; $[S]$ : substrate concentration 


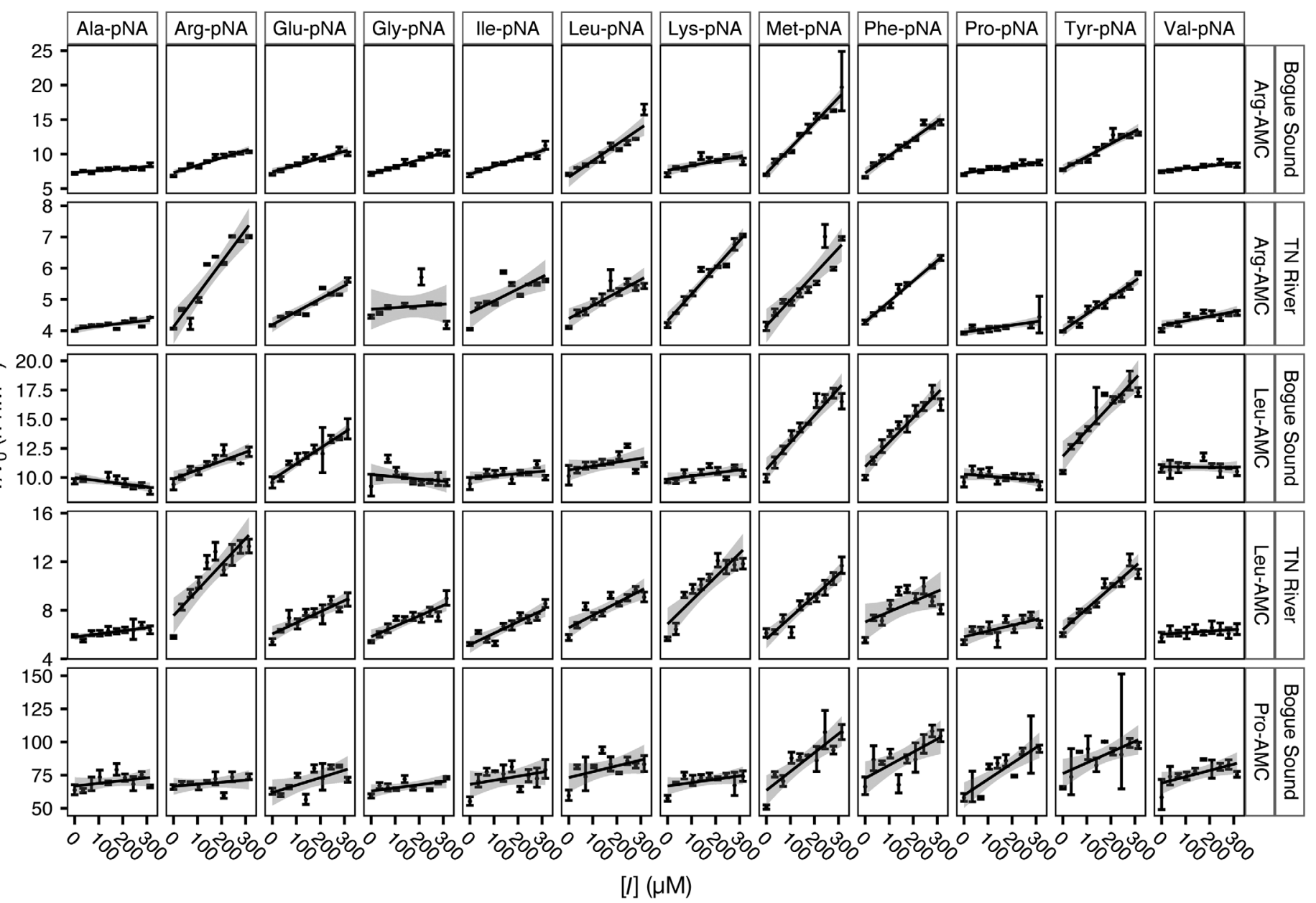

Fig. 4. $1 / V_{0}$ versus $[I]$ for each of 12 amino acid $p$-nitroanilide compounds (Xaa-pNA) at each site (TN: Tennessee). Error bars on the points represent the standard error of the $v_{0}$ estimate. Gray bands represent $95 \%$ confidence intervals for the fit of $1 / v_{0}$ versus $[I]$

\section{Effect of DMSO on enzyme kinetics}

At DMSO concentrations of $4 \%$ or less, DMSO concentration had no effect on $K_{\mathrm{m}}$ ( $\mathrm{p}>0.01$; Fig. 5); $5 \%$ DMSO markedly increased $K_{\mathrm{m}}$. DMSO at lower concentrations had a significant but weak effect on observed $V_{\text {max }}$ : relative to a solution with no DMSO, each additional percentage point of DMSO concentration decreased $V_{\max }$ by $6 \pm 1.8$ percentage points. The $V_{\max }$ estimation at $5 \%$ DMSO concentration was less reliable than the others, because only 2 data points in the saturation curve were measured at concentrations above $K_{\mathrm{m}}$.

\section{Patterns of competitive inhibition}

$K_{\mathrm{I}} / K_{\mathrm{I}, \mathrm{ref}}$ indicates the extent to which an enzyme that hydrolyzes the reference Yaa-AMC also hydro- lyzes a given Xaa-pNA: a low $K_{\mathrm{I}} / K_{\mathrm{I}, \mathrm{ref}}$ indicates a promiscuous enzyme, while a high $K_{\mathrm{I}} / K_{\mathrm{I} \text {,ref }}$ indicates a more substrate-specific enzyme. $K_{\mathrm{I}} / K_{\mathrm{I}, \text { ref }}$ values ranged from 0.15 to 22.2, and varied as a function of both Xaa-pNA and Yaa-AMC, and location (Fig. 6). In many cases, the Xaa-pNA with the lowest $K_{\mathrm{I}} / K_{\mathrm{I} \text {,ref }}$ was not the homologous residue. For instance, in Bogue Sound, 5 Xaa-pNA substrates (Arg-pNA, GlupNA, Met-pNA, Phe-pNA, and Pro-pNA) were better competitors for enzymes hydrolyzing Leu-AMC than was Leu-pNA. Arg-AMC in the Tennessee River was the only example for which the homologous residue displayed the lowest $K_{\mathrm{I}}$. For Pro-AMC in the Tennessee River, Pro-pNA displayed the second-lowest $K_{\mathrm{I}}$.

There was no clear relationship between amino acid R-group chemistry and peptidase affinity. Neither R group hydrophobicity, as quantified by Kyte \& Doolittle (1982), nor the absolute value of the difference between inhibitor R group hydrophobicity and 

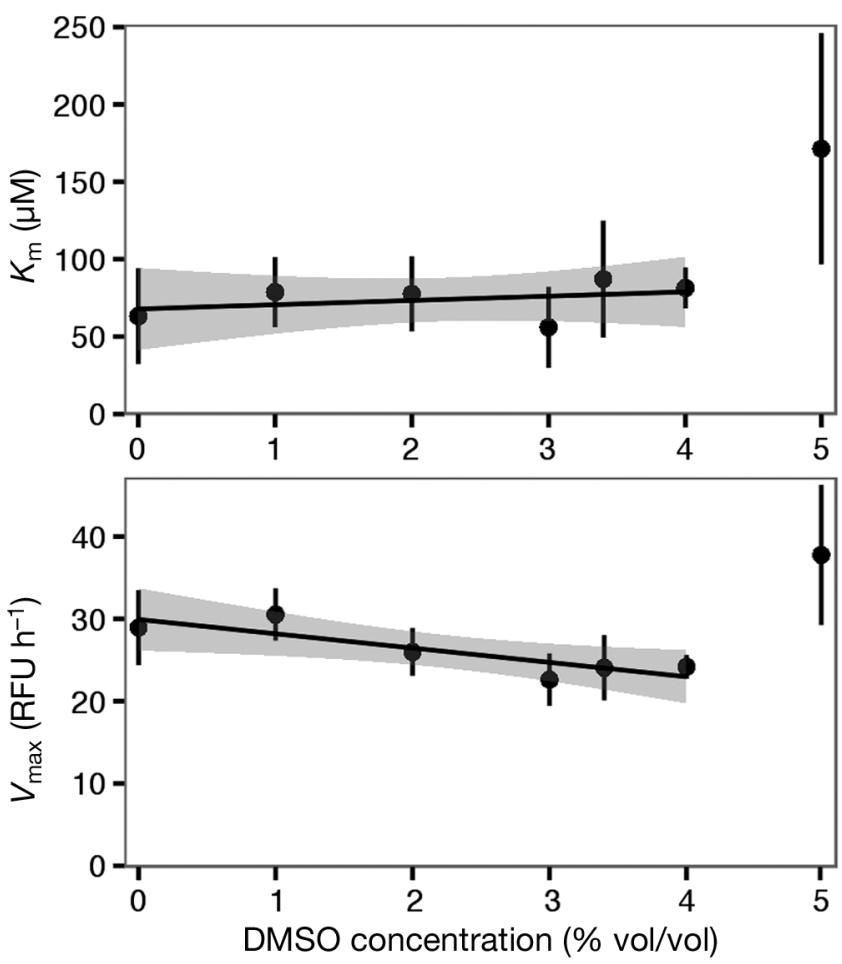

Fig. 5. Effect of dimethyl sulfoxide concentration on kinetics of natural peptidases in the Tennessee River. $v_{\max }$ : maximum theoretical hydrolysis rate; $K_{\mathrm{m}}$ : half-saturation constant RFU: relative fluorescence units. Error bars represent $95 \%$ confidence intervals of the estimate of $K_{\mathrm{m}}$ or $V_{\max }$. Shaded bands represent $95 \%$ confidence intervals of the linear fit

the hydrophobicity of the homologous R group was significantly correlated to $K_{\mathrm{I}} / K_{\mathrm{I}, \mathrm{ref}}$, irrespective of whether each Yaa-AMC substrate and location was considered separately ( $\mathrm{n}=12, \mathrm{p}>0.05$ for each relationship) or the entire data set was considered as a whole ( $\mathrm{n}=60, \mathrm{p}=0.35$ ). $\mathrm{R}$ group functional class (acidic polar, neutral polar, basic polar, or neutral nonpolar) did not predict $K_{\mathrm{I}} / K_{\mathrm{I} \text {,ref }}$ either (ANOVA using log-transformed $K_{\mathrm{I}}: \mathrm{p}=0.21$, df $=56$ ).

Three interrelated factors did predict $K_{\mathrm{I}} / K_{\mathrm{I}, \mathrm{ref}}$ for specific amino acids. $K_{\mathrm{I}} / K_{\mathrm{I}, \text { ref }}$ was negatively related to degradation index (DI) loading (Spearman's $\rho=-0.35$, $\mathrm{p}=0.013, \mathrm{n}=50$; for this analysis we used the loadings reported by Dauwe et al. 1999). For asparagine and glutamine, we used the loadings reported for aspartic acid and glutamic acid, respectively, since the sample hydrolysis protocol used by Dauwe and colleagues deaminates the former 2 residues to produce the latter 2 residues. $K_{\mathrm{I}} / K_{\mathrm{I}, \mathrm{ref}}$ was also negatively correlated to residue molecular weight (Spearman's $\rho=-0.60$, $\mathrm{p}<1 \times 10^{-6}, \mathrm{n}=60$ ) and positively correlated to free energy of complete oxidation to $\mathrm{CO}_{2}\left(\Delta G_{\mathrm{r}}\right.$ i Amend \& Shock 1998) under typical seawater conditions (Spearman's $\rho=0.38, p=0.0031, n=60$; Fig. 7).

\section{DISCUSSION}

At both sites, peptidases hydrolyzing Yaa-AMC displayed higher affinity (lower $K_{\mathrm{I}} / K_{\mathrm{I}, \mathrm{ref}}$ ) for some non-homologous amino acids than they did for the homologous residue (Fig. 6), indicating that extracellular aminopeptidases at each site are substrate unspecific. This result complicates the interpretation of enzyme assays based on small-substrate analogs. For instance, in this study, measurements of uninhibited Leu-AMC hydrolysis rates primarily represented the activities of enzymes that preferentially hydrolyze N-terminal arginine, methionine, and tyrosine, among other residues. Those enzymes are therefore arginyl-, methionyl-, or tyrosyl-aminopeptidases (or others), not leucyl-aminopeptidase.

A similar mismatch between the presumed and actual identities of enzymes hydrolyzing N-terminal leucine has previously been reported (Christian \& Karl 1998). Addition of $80 \mu \mathrm{M}$ L-Leu dimers to surface seawater samples taken near the Antarctic Peninsula only reduced the hydrolysis rate of the aminopeptidase substrate L-leucyl-napthylamine (35 $\mu \mathrm{M})$ by $10 \%$, implying that the enzymes hydrolyzing Lleucyl-napthylamine interacted only weakly with leucine dimers. Interestingly, dimers of L-Met, L-Glu, and L-Gly in that study inhibited hydrolysis of Lleucyl-napthylamine at least as much as L-Leu dimers did, consistent with the data presented here that enzymes other than leucyl-aminopeptidase were primarily responsible for hydrolyzing N-terminal Leu enzyme substrates.

\section{Influences on substrate affinity}

The correlations between $K_{\mathrm{I}} / K_{\mathrm{I} \text {,ref }}$ and molecular weight, Dauwe DI loading, and $\Delta G_{\mathrm{r}}$ for oxidation to $\mathrm{CO}_{2}$ suggest that substrate specificities of peptidases may be subject to selective pressure and may have geochemical consequences.

DI is an index based on principal components analysis of total hydrolysable amino acid content (THAA) in marine sediments, which describes the diagenetic status of organic matter in terms of the relative proportions of amino acids in the sample. According to this index, each amino acid is assigned a loading. Negative DI loadings indicate amino acids that are relatively more abundant in fresh organic matter, whereas positive DI loadings indicate amino acids that are relatively enriched in more degraded organic matter. While DI does not accurately describe amino acid diagenesis everywhere it has been 


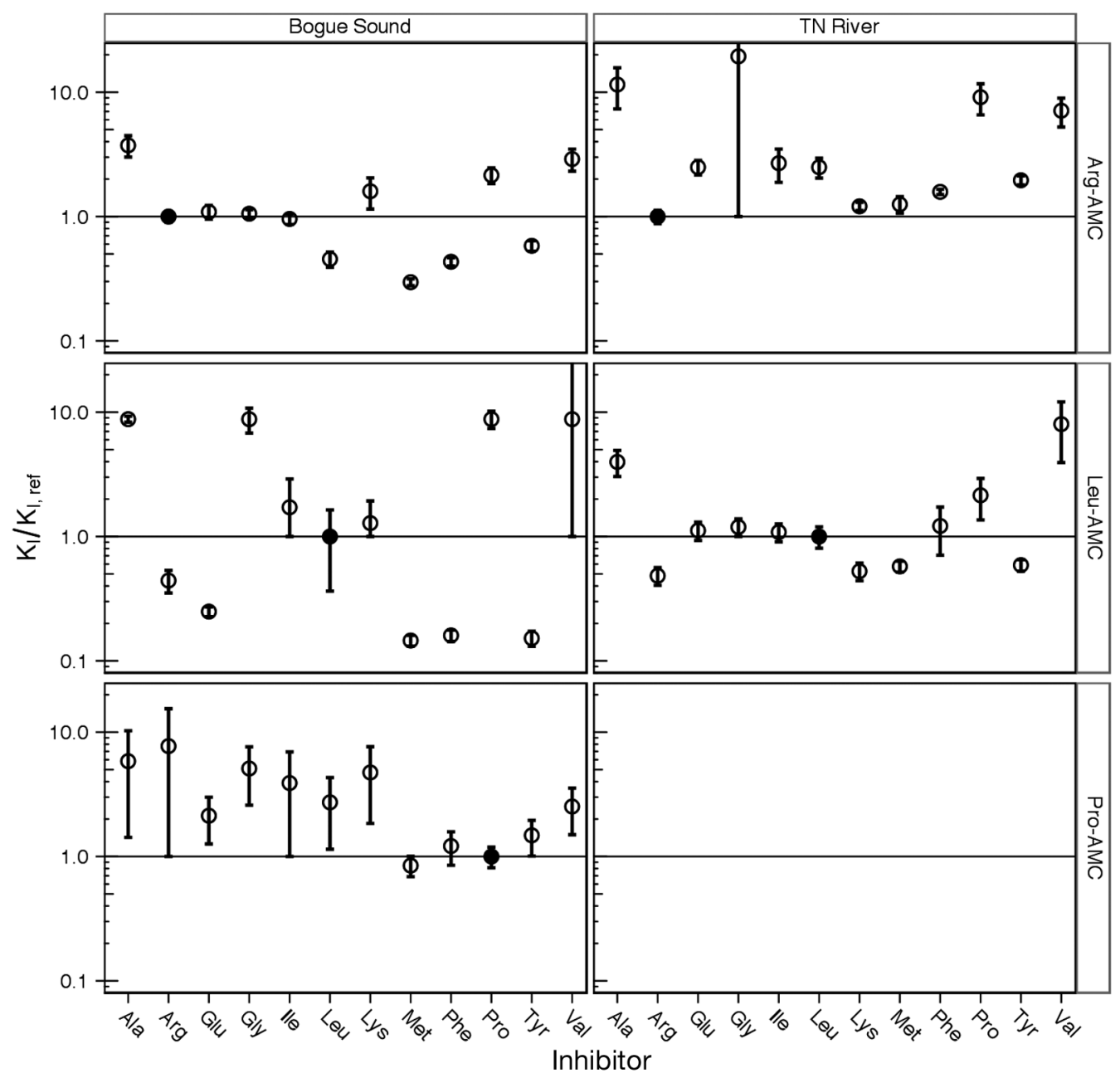

Fig. 6. Affinities of Xaa-pNA competitors for enzymes hydrolyzing Arg-AMC, Leu-AMC, and Pro-AMC in the Tennessee (TN) River at Knoxville, TN, and in Bogue Sound, NC. Black symbols indicate the Xaa-pNA inhibitor homologous to the Yaa-AMC substrate. Error bars represent the standard error of the estimate of $K_{\mathrm{I}} / K_{\mathrm{I}, \text { ref, }}$ based on Eq. (3)

applied (Davis et al. 2009), it is applicable to a wide range of environments (Burdige 2006).

The correlations between $K_{\mathrm{I}} / K_{\mathrm{I}, \mathrm{ref}}$ and DI loading, molecular weight, and $\Delta G_{\mathrm{r}}$ (Fig. 7) indicated that those residues for which a broader range of peptidases have higher affinity are removed more rapidly from bulk organic matter during early diagenesis. Aminopeptidases employ a wide variety of mechanisms to bind substrate at the active site (Wilce et al. 1998). We were unable to identify a simple biochemical mechanism that could cause this trend. We propose that DI loadings may be driven in part by patterns of peptidase substrate specificity. The reason for this could be ecological: microorganisms might produce extracellular peptidases with higher specificity for larger amino acids because they would provide more carbon (and in some cases nitrogen) or free energy per peptide bond cleaved than would smaller amino acids. These results suggest a scenario in which extracellular peptidases are under selective pressure to be more active towards larger amino acids, which would provide more free energy upon metabolism. The cumulative result is that larger amino acids with more negative $\Delta G_{\mathrm{r}}$ values are preferentially removed from sediments, thus giving rise to the patterns of amino acid diagenesis that have been quantified using DI.

\section{Implications for interpreting enzyme assays}

The general reliance of the aquatic ecology community on Leu-AMC to assay a broad class of enzymes potentially presents an unrealistically nar- 

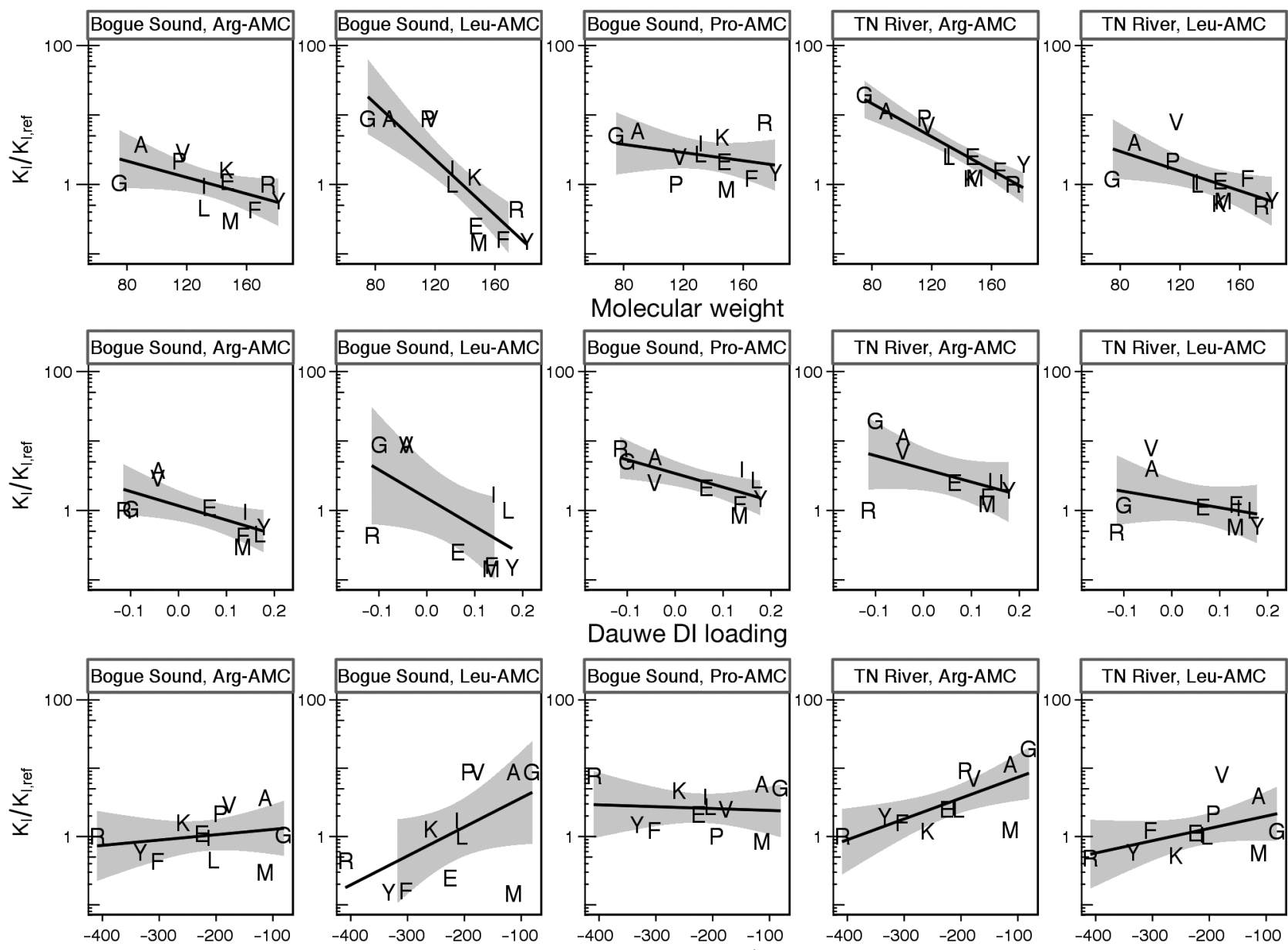

Fig. 7. Relationships between affinity $\left(K_{\mathrm{I}} / K_{\mathrm{I}, \mathrm{ref}}\right)$ and molecular weight, degradation index (DI; sensu Dawes et al. 1999) loading, and free energy of complete oxidation to $\mathrm{CO}_{2}$ and $\mathrm{NH}_{4}{ }^{+}\left(\Delta G_{\mathrm{r}}\right)$. The dark line represents a linear least-squares regression; the shaded area represents the $95 \%$ confidence interval for that regression

row view of the types of extracellular peptidases present in aquatic environments. The few studies in which fluorogenic substrates other than Leu-AMC have been applied (e.g. Obayashi \& Suzuki 2005, 2008a, Steen \& Arnosti 2013) show that a diverse but variable set of peptidases is active in aquatic environments. Use of Leu-AMC as a proxy for total peptidase is therefore potentially misleading; Leu-AMC hydrolysis accounts for a variable (Steen \& Arnosti 2013) and often small (Obayashi \& Suzuki 2005, 2008b, Bong et al. 2013) fraction of total peptidolytic potential. The results here demonstrate that LeuAMC hydrolysis does not even necessarily reflect the activity of leucyl-aminopeptidase in a given environment. For instance, the enzymes hydrolyzing Leu$\mathrm{AMC}$ at Bogue Sound were better described as arginine-, glutamine-, methionine-, phenylalanine-, and tyrosine-aminopeptidases, since they were more spe- cific for those N-terminal residues than they were for leucine. Previous studies of peptidase activities in aquatic environments should be interpreted in light of the fact that many peptidases other than leucylaminopeptidase likely contributed to the activity of 'leucyl-aminopeptidase' as assayed by Leu-AMC.

In some studies, $V_{\max }$ for Leu-AMC hydrolysis is interpreted as a quantitative proxy of the total peptidolytic potential of a community (Kellogg et al. 2011). The results presented here show that approach to be invalid. In other studies, changes in $V_{\max }$ for LeuAMC hydrolysis are used as a response variable to assess changes in the peptidolytic potential of microbial communities among experimental treatments, locations, or time points (Allison et al. 2012). When enzyme activities are used in this way, the fact that multiple peptidases hydrolyze Leu-AMC is helpful, in the sense that Leu-AMC hydrolysis rates integrate 
the activities of a wide range of specific enzymes. Leu-AMC hydrolysis therefore presents a more complete picture of community protein demand than it would if aquatic peptidases were highly substrate specific and Leu-AMC were only hydrolyzed by leucyl-aminopeptidase.

Primary producers synthesize thousands of distinct proteins and peptide-containing molecules. Although these proteins are typically measured in bulk after protein hydrolysis, e.g. as 'total hydrolysable amino acids' (Lindroth \& Mopper 1979), their degree of bioavailability depends on their specific chemical properties: primary, secondary and tertiary structure, post-translational modifications, and physical associations (for example, sorption to mineral surfaces, incorporation in complexes, etc.; Arnosti 2011). The substrate specificities of aquatic peptidases therefore determine the number of individual peptidases a community must produce to efficiently mineralize available proteins. The results presented here demonstrate that multiple extracellular peptidases are required to hydrolyze the full range of proteins that might exist in aquatic environments. This means that the ability to consume a specific subset of proteinaceous organic matter may define an ecological niche, and microbial taxa which express different peptidases inhabit different ecological niches (Zimmerman et al. 2013). To gain a better mechanistic understanding of the process by which heterotrophic microbial communities mineralize proteinaceous organic matter, a better understanding of the specific biochemical properties of microbial peptidases is required.

Acknowledgements. The authors thank Mike Piehler and Aron Stubbins for generously offering laboratory space at the University of North Carolina Institute of Marine Sciences and Skidaway Institute of Oceanography, respectively, and Karen Lloyd for laboratory assistance. Jeffery Becker offered helpful comments on the manuscript. We thank 3 anonymous reviewers for their suggestions on the manuscript. A.D.S. was supported by grants from the Center for Dark Energy Biosphere Investigations and NSF grant OCE1061352. K.H.M. was supported through a NSF REU site award (DBI-1156644) to S.W.W.

\section{LITERATURE CITED}

Allison SD, Chao Y, Farrara JD, Hatosy S, Martiny A (2012) Fine-scale temporal variation in marine extracellular enzymes of coastal southern California. Frontiers Microbiol 3:301

> Amend JP, Shock EL (1998) Energetics of amino acid synthesis in hydrothermal ecosystems. Science 281: 1659-1662

Arnosti C (1996) A new method for measuring polysaccharide hydrolysis rates in marine environments. Org Geochem 25:105-115
Arnosti C (2011) Microbial extracellular enzymes and the marine carbon cycle. Ann Rev Mar Sci 3:401-425

Arnosti C, Bell C, Moorhead D, Sinsabaugh RL and others (2014) Extracellular enzymes in terrestrial, freshwater, and marine environments: system variability and common needs. Biogeochemistry 117:5-21

Arrieta J, Herndl G (2002) Changes in $\beta$-glucosidase diversity during a coastal phytoplankton bloom. Limnol Oceanogr 47:594-599

> Bailey VL, Fansler SJ, Smith JL, Bolton H Jr (2011) Reconciling apparent variability in effects of biochar amendment on enzyme activities by assay optimization. Soil Biol Biochem 43:296-301

> Bong CW, Obayashi Y, Suzuki S (2013) Succession of protease activity in seawater and bacterial isolates during starvation in a mesocosm experiment. Aquat Microb Ecol 69:33-46

Burdige DJ (2006) Geochemistry of marine sediments. Princeton University Press, Princeton, NJ

> Christian JR, Karl DM (1998) Ectoaminopeptidase specificity and regulation in Antarctic marine pelagic microbial communities. Aquat Microb Ecol 15:303-310

> Dauwe B, Middelburg JJ, Herman PMJ, Heip CHR (1999) Linking diagenetic alteration of amino acids and bulk organic matter reactivity. Limnol Oceanogr 44: 1809-1814

> Davis J, Kaiser K, Benner R (2009) Amino acid and amino sugar yields and compositions as indicators of dissolved organic matter diagenesis. Org Geochem 40:343-352

- German DP, Weintraub MN, Grandy AS, Lauber CL, Rinkes ZL, Allison SD (2011) Optimization of hydrolytic and oxidative enzyme methods for ecosystem studies. Soil Biol Biochem 43:1387-1397

Hoppe HG (1983) Significance of exoenzymatic activities in the ecology of brackish water: measurements by means of methylumbelliferyl-substrates. Mar Ecol Prog Ser 11: 299-308

> Kellogg C, Carpenter S, Renfro A, Sallon A, Michel C, Cochran J, Deming J (2011) Evidence for microbial attenuation of particle flux in the Amundsen Gulf and Beaufort Sea: elevated hydrolytic enzyme activity on sinking aggregates. Polar Biol 34:2007-2023

> Kyte J, Doolittle RF (1982) A simple method for displaying the hydropathic character of a protein. J Mol Biol 157: 105-132

Leskovac V (2003) Comprehensive enzyme kinetics. Kluwer Academic Publishers, New York, NY

> Lindroth P, Mopper K (1979) High performance liquid chromatographic determination of subpicomole amounts of amino acids by precolumn fluorescence derivatization with o-phthaldialdehyde. Anal Chem 51:1667-1674

> Liu Z, Kobiela ME, McKee GA, Tang T, Lee C, Mulholland MR, Hatcher PG (2010) The effect of chemical structure on the hydrolysis of tetrapeptides along a river-to-ocean transect: AVFA and SWGA. Mar Chem 119:108-120

Nomoto M, Narahashi Y, Murakami M (1960) A proteolytic enzyme of Streptomyces griseus. VII. Substrate specificity of Streptomyces griseus protease. J Biochem 48: 906-918

Obayashi Y, Suzuki S (2005) Proteolytic enzymes in coastal surface seawater: significant activity of endopeptidases and exopeptidases. Limnol Oceanogr 50:722-726

Obayashi Y, Suzuki S (2008a) Adsorption of extracellular proteases in seawater onto filters during size fractionation. J Oceanogr 64:367-372 
Obayashi Y, Suzuki S (2008b) Occurrence of exo- and endopeptidases in dissolved and particulate fractions of coastal seawater. Aquat Microb Ecol 50:231-237

Odagami T, Morita J, Takama K, Suzuki S (1994) Substrate specificities of extracellular proteases produced by marine putrefactive bacteria, Shewanella putrefaciens and Alteromonas haloplanktis. Lett Appl Microbiol 18:50-52

Pantoja S, Lee C (1999) Peptide decomposition by extracellular hydrolysis in coastal seawater and salt marsh sediment. Mar Chem 63:273-291

Pantoja S, Lee C, Maracek JF (1997) Hydrolysis of peptides in seawater and sediment. Mar Chem 57:25-40

Pantoja S, Rossel P, Castro R, Cuevas LA, Daneri G, Córdova C (2009) Microbial degradation rates of small peptides and amino acids in the oxygen minimum zone of Chilean coastal waters. Deep-Sea Res II 56:1055-1062

R Core Team (2013) R: a language and environment for statistical computing. R Foundation for Statistical Computing, Vienna

Steen AD, Arnosti C (2011) Long lifetimes of $\beta$-glucosidase, leucine aminopeptidase and phosphatase in Arctic seawater. Mar Chem 123:127-132

Steen AD, Arnosti C (2013) Extracellular peptidase and carbohydrate hydrolase activities in an Arctic fjord (Smeerenburgfjord, Svalbard). Aquat Microb Ecol 69:93-99

Editorial responsibility: Hans-Georg Hoppe, Kiel, Germany
Steen AD, Ziervogel K (2012) Comment on the review by German et al. (2011) 'Optimization of hydrolytic and oxidative enzyme methods for ecosystem studies' [Soil Biology \& Biochemistry 43:1387-1397]. Soil Biol Biochem 48:196-197

Steen AD, Arnosti C, Ness L, Blough NV (2006) Electron paramagnetic resonance spectroscopy as a novel approach to measure macromolecule-surface interactions and activities of extracellular enzymes. Mar Chem 101: 266-276

Thao NV, Obayashi Y, Yokokawa T, Suzuki S (2014) Coexisting protist-bacterial community accelerates protein transformation in microcosm experiments. Front Mar Sci 1:69

> Wang G, Post WM, Mayes MA (2012) Parameter estimation for models of ligninolytic and cellulolytic enzyme kinetics. Soil Biol Biochem 48:28-38

Wickham H (2009) ggplot2: elegant graphics for data analysis. Springer, New York, NY

- Wilce MCJ, Bond CS, Dixon NE, Freeman HC, Guss JM, Lilley PE, Wilce JA (1998) Structure and mechanism of a proline-specific aminopeptidase from Escherichia coli. Proc Natl Acad Sci USA 95:3472-3477

Zimmerman AE, Martiny AC, Allison SD (2013) Microdiversity of extracellular enzyme genes among sequenced prokaryotic genomes. ISME J 7:1187-1199

Submitted: February 3, 2014; Accepted: April 13, 2015 Proofs received from author(s): July 13, 2015 